\title{
Paracoccidioidomicose com comprometimento do sistema nervoso central: revisão sistemática da literatura
}

\author{
Paracoccidioidomycosis compromising the \\ central nervous system: a systematic review of the literature
}

\author{
Vinicius Sousa Pietra Pedroso ${ }^{1,2}$, Márcia de Carvalho Vilela ${ }^{1,2}$, \\ Enio Roberto Pietra Pedroso ${ }^{1,3}$ e Antônio Lúcio Teixeira ${ }^{1,2,3}$
}

\begin{abstract}
RESUMO
Este trabalho é o primeiro a realizar uma revisão sistemática dos casos de neuroparacoccidioidomicose disponíveis na literatura. Foram encontrados 257 casos em 81 trabalhos pesquisados pelo MEDLINE e LILACS, com maior número de publicações após as décadas de 1970-1980. Aproximadamente, 93\% dos pacientes eram homens, principalmente lavradores, com idade média de 43 anos. 0 quadro caracterizou-se por sintomatologia motora ou de hipertensão intracraniana. A forma crônica pseudotumoral predominou. 0 período médio de evolução foi de 4,9 meses. As lesões foram principalmente supratentoriais $(66,8 \%)$, localizando-se nos lobos frontais e parietais. A biópsia determinou o diagnóstico em 57,2\% dos casos e utilizaram-se métodos de neuroimagem em 64,6\% deles. Houve grande associação com a forma pulmonar da doença (59,1\%). A mortalidade foi de 44,1\% e 50,1\% dos sobreviventes evoluíram com sequelas, principalmente motoras. Assim, deve-se considerar a neuroparacoccidioidomicose no diagnóstico diferencial dos processos expansivos e meningoencefalíticos do sistema nervoso central para se estabelecer tratamento precoce e evitar seqüelas incapacitantes.
\end{abstract}

Palavras-chaves: Paracoccidioidomicose. Infecções fúngicas. Neuroparacoccidioidomicose. Sistema nervoso central. Revisão sistemática.

\section{ABSTRACT}

This study is the first systematic review of cases of neuroparacoccidioidomycosis available in the literature. Through searches in the MEDLINE and LILACS databases, 257 cases were found in 81 published studies, mainly after the 1970s-1980s. Approximately $93 \%$ of the patients were men, especially farm laborers, with a mean age of 43 years. The characteristic symptoms were motor deficits or intracranial hypertension. The chronic pseudotumoral form predominated. The mean period of evolution was 4.9 months. The lesions were mainly supratentorial $(66.8 \%)$, located in the frontal and parietal lobes. The diagnosis was determined by biopsy in $57.2 \%$ of the cases and neuroimaging methods were used in $64.6 \%$ of them. A large proportion of the cases were associated with the pulmonary form of the disease $(59.1 \%)$. The mortality rate was $44.1 \%$, and $50.1 \%$ of the survivors developed sequelae, especially motor impairment. Thus, neuroparacoccidioidomycosis should be considered in the differential diagnosis for expansive and meningoencephalitic processes in the central nervous system, in order to establish early treatment and to avoid disabling sequelae.

Key-words: Paracoccidioidomycosis. Fungal infection. Neuroparacoccidioidomycosis. Central nervous system. Systematic review.

A paracoccidioidomicose (PCM), infecção sistêmica causada pelo fungo Paracoccidioides brasiliensis, é considerada a mais importante micose profunda da América Latina ${ }^{48}$. Pode ser considerada, ainda, um problema de saúde pública em função de seus elevados custos sociais e econômicos decorrentes não apenas

1. Curso de Pós-Graduação em Ciências da Saúde: Infectologia e Medicina Tropical, Faculdade de Medicina, Universidade Federal de Minas Gerais, Belo Horizonte, MG. 2. Laboratório de Imunofarmacologia, Departamento de Bioquímica e Imunologia, Instituto de Ciências Biológicas, Universidade Federal de Minas Gerais, Belo Horizonte, MG. 3. Departamento de Clínica Médica, Faculdade de Medicina, Universidade Federal de Minas Gerais, Belo Horizonte, MG

Apoio financeiro: Conselho Nacional de Desenvolvimento Científico e Tecnológico (CNPq).

Endereço para correspondência: Dr. Antônio Lúcio Teixeira. Departamento de Clínica Médica/FM/UFMG. Av. Prof. Alfredo Balena 190, Santa Efigênia, 30130-100 Belo Horizonte, MG.

Tel: 5531 9957-5234

e-mail: altexr@gmail.com

Recebido para publicação em 24/03/2009

Aceito em 08/10/2009 da doença em atividade, mas também das freqüentes seqüelas, motivo comum de incapacitação para o trabalho ${ }^{1549}$.

Afeta indivíduos em todas as faixas etárias a partir dos dois anos de idade, mas há marcante predomínio entre os 30 e os 50 anos de vida, período que compreende o auge da capacidade produtiva dos pacientes. A incidência é a mesma para ambos os sexos até a puberdade, entretanto, na vida adulta, torna-se mais freqüente entre os indivíduos do sexo masculino, em proporção de $11: 1^{22}$.

A doença pode manifestar-se de inúmeras maneiras e $o$ fungo pode causar lesões em virtualmente qualquer órgão. De modo geral, os pulmões são os órgãos mais frequentemente acometidos, mas lesões cutâneas, de mucosas, linfonodais e adrenais também são comuns. Nas últimas décadas, com o surgimento de novos métodos diagnósticos, especialmente os de imagem, constatou-se que 0 envolvimento do sistema nervoso central (SNC) ébem mais frequente do que se admitia, ocorrendo em torno de $12,5 \%$ dos casos de PCM e chegando, em alguns estudos, até $36 \%$ dos $\operatorname{casos}^{62}$. 
A neuroparacoccioidomicose (NPCM) é a infecção do SNC pelo fungo $0^{54} \mathrm{e}$ as informações disponíveis na literatura acerca dessa forma ainda são notavelmente fragmentadas e baseadas em relatos de casos únicos ou de pequenas séries de casos. Assim, 0 presente trabalho objetiva realizar uma revisão sistemática da NPCM.

\section{MATERIAL E MÉTODOS}

Foram utilizados os bancos de dados MEDLINE e LILACS para a realização de pesquisas iniciais a partir dos termos de busca neuroparacoccidioidomycosis, central nervous system paracoccidioidomycosis, neurological paracoccidioidomycosis. Em seguida, foram realizadas buscas de todas as referências bibliográficas dos estudos obtidos inicialmente na tentativa de conseguir o maior número de publicações disponíveis no assunto. As informações demográficas e clínicas dos relatos contidos em todos os trabalhos foram reunidas e sistematizadas em um banco de dados gerado no programa SPSS 12.0 a fim de viabilizar análise estatística.

\section{RESULTADOS}

Foram encontrados 81 trabalhos publicados no período compreendido entre os anos de 1919 a 2007, os quais relatavam 266 casos $^{12345678910111213141617181920212223242526272829313233343536}$ 3738394041424344454647505152535556575859606163646566676869707172737576 777879808183848586878889 . Após cuidadosa análise de todos os casos, foram identificados nove pacientes cujos relatos apareciam em mais de um trabalho, de modo que foram analisadas as histórias de 257 pacientes com NPCM. A natureza dos trabalhos variou desde relatos de casos únicos até séries de casos, havendo grande variedade na quantidade de informações disponíveis entre os diversos estudos. Dentre estes, o estudo que concentrou a maior casuística foi o de Almeida e cols ${ }^{2}$, em que foram analisados aspectos clínicos e laboratoriais de 24 pacientes com NPCM. A distribuição dos trabalhos ao longo do tempo é representada na Figura 1.
A distribuição entre os sexos foi notadamente desigual $(\mathrm{p}=0,005$; teste $\mathrm{t}$ para amostras independentes), com aproximadamente $93 \%$ dos pacientes pertencentes ao sexo masculino. A idade média foi de aproximadamente 43 anos $( \pm 9,57)$, variando de 12 a 66 anos. A média de idade foi diferente de acordo com o gênero, sendo de aproximadamente 44 anos entre os pacientes do sexo masculino e de 34 anos entre as mulheres ( $\mathrm{p}=0,021$; teste $\mathrm{t}$ para amostras independentes). Com relação à distribuição dos pacientes de acordo com a atividade profissional, 39 trabalhos traziam essa informação, com um total de 85 casos relatados, de modo que 56\% deles eram lavradores, $12 \%$ eram motoristas, $4 \%$ trabalhavam na construção civil, $2 \%$ exerciam atividades de faxineiro e $26 \%$ possuíam alguma outra ocupação.

A Tabela 1 apresenta a frequência de observação dos principais sinais e sintomas apresentados por 221 pacientes durante o curso da doença, destacando-se os sinais motores e os de hipertensão intracraniana.

Do total de trabalhos, 13 traziam informações sobre o tempo de evolução da doença, definido como o período de tempo entre o início do quadro neurológico e a procura por atendimento médico, com um total de 38 casos. 0 tempo médio de evolução observado foi de 4,9 meses variando de alguns poucos dias a até 18 meses.

Aproximadamente, 98,3\% dos pacientes apresentavam a forma crônica da PCM, enquanto 1,7\% apresentavam a forma aguda/

TABELA 1

Principais síndromes neurológicas observadas em relatos de 221 casos de neuroparacoccidioidomicose.

\begin{tabular}{lc}
\hline Sinais e sintomas & Porcentagem \\
\hline Sinais e sintomas motores & 49,8 \\
Sinais e sintomas sugestivos de hipertensão intracraniana & 48,9 \\
Crises epilépticas & 24,9 \\
Sinais e sintomas cerebelares & 22,2 \\
Sinais e sintomas cognitivos & 21,3 \\
Sintomas sensitivos & 18,1 \\
Sinais meníngeos & 8,6 \\
Sinais e sintomas medulares & 8,1 \\
\hline
\end{tabular}

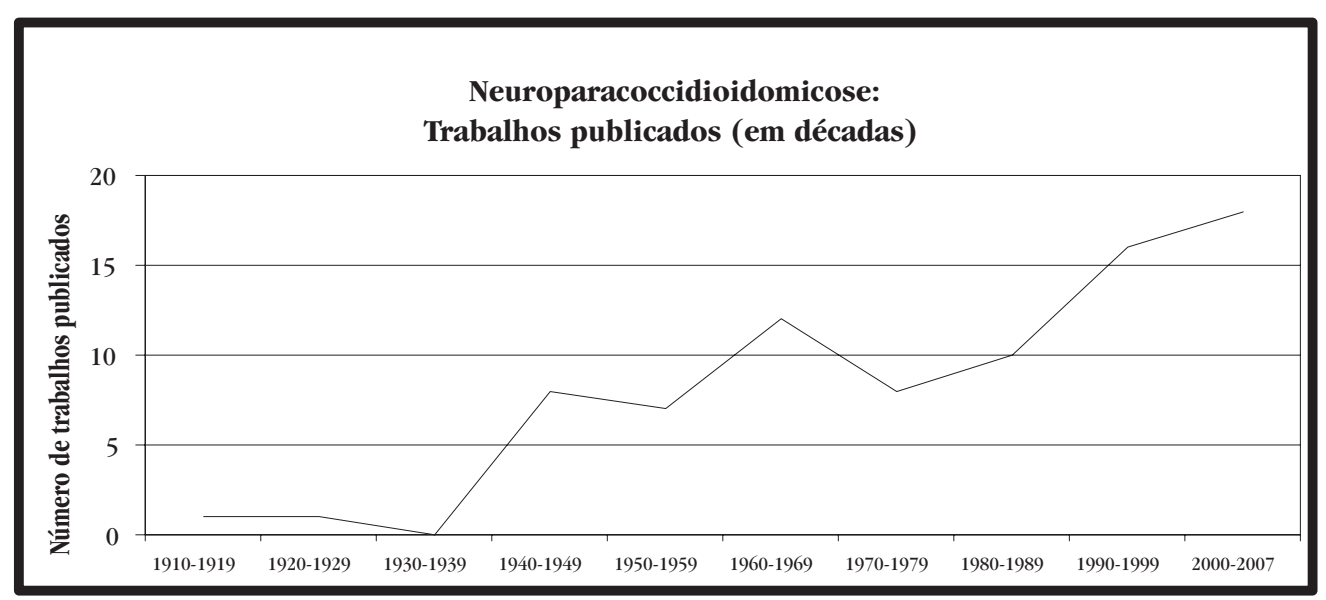

FIGURA 1

Distribuição das 81 publicações sobre neuroparacoccidioidomicose ao longo das décadas desde sua primeira descrição. 
subaguda, dado apresentado por 80 trabalhos, totalizando 252 casos. A forma pseudotumoral da NPCM foi observada em $89,4 \%$ dos casos, consistindo em abscessos, granulomas, nódulos ou cistos intraparenquimatosos, a passo que $10,6 \%$ exibiam a forma meningoencefalítica, caracterizada por processo inflamatório crônico da base do encéfalo, que pode ocasionar sintomatologia radicular ou mielítica bastante semelhante à meningoencefalite tuberculosa ${ }^{14}$, de acordo com a descrição de 251 casos presentes em 79 trabalhos.

A Tabela 2 apresenta a frequência de observação de lesões paracoccidioidomicóticas em sítios anatômicos do sistema nervoso central em 222 casos. As lesões nos hemisférios cerebrais, sobretudo lobos frontal e parietal, e no cerebelo foram as mais freqüientes.

\section{TABELA 2}

Principais regiões de localização de lesões no sistema nervoso central relatadas em 222 casos de neuroparacoccidioidomicose.

\begin{tabular}{lc}
\hline Distribuição das lesões & Porcentagem \\
\hline Supratentorial & 66,8 \\
Hemisférios cerebrais & 47,6 \\
Frontal & 13,1 \\
Parietal & 17,0 \\
Temporal & 7,9 \\
Occipital & 8,7 \\
Núcleos da base & 3,5 \\
Tálamo & 6,6 \\
Infratentorial & 42,4 \\
Cerebelo & 28,8 \\
Tronco & 14,4 \\
Mesencéfalo & 3,5 \\
Ponte & 3,5 \\
Bulbo & 2,2 \\
Medular & 9,2 \\
Cervical & 2,6 \\
Torácica & 4,8 \\
Lombar & 0,0 \\
Sacral & 0,0 \\
Ventrículos & 2,2 \\
\hline &
\end{tabular}

0 diagnóstico foi estabelecido através da biópsia em 57,2\% dos 257 casos; por métodos sorológicos em $25,7 \%$ deles; por cultura em 23,3\%. 0 estudo do líquor contribuiu para definição do diagnóstico em 16,3\% dos casos, sendo 16,3\% dos casos com a utilização de métodos imunológicos, 1,9\% por meio da microscopia direta e 1,2\% pela cultura. Aproximadamente, $53,7 \%$ do total de pacientes realizaram exames tomográficos do encéfalo e 10,9\%, ressonância magnética. Cerca de 71,2\% do total de pacientes com NPCM apresentaram alterações à radiografia torácica.

A Tabela 3 apresenta a distribuição do acometimento simultâneo de sítios externos ao sistema nervoso central observada entre 216 pacientes com NPCM, sendo o pulmão o órgão mais afetado.
TABELA 3

Frequência de comprometimento de outros órgãos relatada em 216 casos de neuroparacoccidioidomicose.

\begin{tabular}{lc}
\hline Associação com PCM em outros sítios & Porcentagem \\
\hline Pulmões & 59,1 \\
Mucosas & 27,2 \\
Pele & 16,3 \\
Linfonodos & 15,9 \\
Adrenais & 10,9 \\
Ossos & 0,4 \\
\hline
\end{tabular}

A Tabela 4 mostra a frequência de utilização dos diferentes esquemas terapêuticos para a NPCM observada entre 179 casos. 0 tempo médio de tratamento descrito foi de 8,2 meses, variando desde a completa falta de tratamento até 30,9 meses de terapia. 0 tratamento cirúrgico caracterizou-se por craniotomia com drenagem de abscesso.

TABELA 4

Esquemas terapêuticos empregados no tratamento de 179 casos de neuroparacoccidioidomicose.

\begin{tabular}{lc}
\hline Esquemas terapêuticos & Porcentagem \\
\hline Sulfametoxazol-Trimetoprim & 36,3 \\
Anfotericina B & 31,3 \\
Antimicrobiano sulfamídico (excluindo SMT-TMP) & 21,2 \\
Cetoconazol & 8,3 \\
Fluconazol & 8,3 \\
Itraconazol & 3,9 \\
Tratamento cirúrgico & 38,5
\end{tabular}

Havia registros sobre o desfecho de 211 casos. Destes, aproximadamente, 44,1\% evoluíram para o óbito. Entre os 118 pacientes sobreviventes, havia registros sobre a evolução de 84 casos. Destes, $50 \%$ permaneceram com algum tipo de seqüela (Tabela 5).

\section{TABELA 5}

Sequelas observadas em relatos de 84 casos de neuroparacoccidioidomicose, dos quais 42 exibiam algum tipo de sequela.

\begin{tabular}{lc}
\hline Tipo de sequela & Porcentagem \\
\hline Sequelas motoras & 28,6 \\
Sequelas sensitivas & 8,3 \\
Convulsões & 4,8 \\
Sequelas medulares & 4,8 \\
Sequelas cognitivas & 2,4 \\
Alterações de marcha & 1,2 \\
\hline
\end{tabular}

Finalmente, foram obtidos dados com relação ao acompanhamento tomográfico de 50 pacientes, observando-se melhora radiológica total em $22 \%$ dos casos, melhora moderada em $72 \%$ deles, melhora leve em $2 \%$ e ausência de modificação das lesões em 4,0\% dos casos. 


\section{DISCUSSÃO}

Este é o primeiro trabalho a realizar uma revisão sistemática dos casos neurológicos de PCM disponíveis na literatura, agregando informações de 257 casos individuais da doença.

A frequência do acometimento do SNC foi inicialmente descrita como rara, possivelmente devido a apresentações assintomáticas, exame neurológico inadequado, falta de testes específicos como tomografia computadorizada (TC) ou ressonância nuclear magnética (RNM) e baixa frequência de estudo do SNC em necropsias ${ }^{52}$. Com os novos métodos diagnósticos que surgiram nas últimas décadas, constatou-se que a NPCM constitui eventualidade bem mais frequiente que se admitia, ocorrendo em torno de 9,9 a 27\% dos casos de PCM, chegando até mesmo a 36\% em estudo necroscópico realizado por Franco e cols ${ }^{30}$. Em trabalho publicado por Hutzer e cols ${ }^{36}, 40$ pacientes com PCM e com exame clínico, neurológico e de líquor normais foram submetidos a TC do encéfalo encontrando-se $12,5 \%$ de positividade para o acometimento do SNC. Desses pacientes, 60\% apresentaram, em épocas diferentes da observada, sintomas neurológicos; fato importante ao se considerar que a taxa de mortalidade da NPCM chega a $53 \%^{22}$, tendo sido observada uma taxa de mortalidade de 44,1\% no presente estudo. A análise dos dados confirma a tendência de aumento do volume de relatos de casos de NPCM ao longo do tempo, principalmente após as décadas de 1970-1980, fato que talvez seja explicado pela evolução dos métodos diagnósticos citados, especialmente os de neuroimagem.

Poucos trabalhos na literatura estabelecem comparações entre pacientes com a forma neurológica e pacientes sem a forma neurológica da PCM. Na maior parte destes trabalhos comparativos, realizam-se somente investigações diagnósticas, comparando-se a detecção de determinados antígenos no soro de pacientes com e sem doença neurológica, ou avaliando-se a capacidade de detecção desses marcadores no líquor ${ }^{74} 81$. Cabe mencionar ainda o trabalho de Almeida e cols ${ }^{3}$, que, a partir da descrição da associação entre PCM e alguns alelos do antígeno leucocitário humano (HLA) ou de histocompatibilidade principal, realizaram um estudo com o objetivo de investigar essa associação na NPCM. Portanto, faltam trabalhos que buscam definir objetivamente aspectos singulares da NPCM em relação à PCM. Isso pode ser, em parte, definido a partir da análise sistemática dos casos de NPCM descritos.

A distribuição dos casos segundo sexo segue o padrão geral observado para a PCM, com predominância no sexo masculino em proporção aproximada a 14:1. A média de idade dos pacientes com a forma neurológica da doença também foi semelhante à média de idade geral observada entre os casos de PCM. Entretanto, de modo interessante, observou-se, entre os casos do sexo feminino, uma tendência ao surgimento da doença em pacientes significativamente mais jovens que entre os do sexo masculino, fato até certo ponto inesperado ao se considerar a relativa proteção feminina ao desenvolvimento da PCM durante o período reprodutivo da vida.
Com relação à ocupação profissional dos indivíduos, foi observada clara predominância do acometimento de lavradores, em concordância com o perfil amplamente atribuído à PCM.

o quadro clínico caracteriza-se principalmente pela ocorrência de sinais e sintomas motores, especialmente alterações na força muscular, associados a sinais e sintomas sugestivos de hipertensão intracraniana, como cefaléia ou papiledema. 0 surgimento de crises convulsivas, sinais e sintomas cerebelares, como dismetria ou distúrbios de marcha, e transtornos mentais como alterações da cognição ou da memória, também foram relativamente comuns. Em três diferentes estudos realizados por Almeida e cols $^{2}$, Elias Jr e cols ${ }^{21}$ e Fagundes-Pereyra ${ }^{22}$, os autores observaram as principais alterações clínicas em 24, 20 e 13 casos de NPCM, respectivamente. As principais manifestações relatadas nos três estudos foram crises epilépticas, déficits motores, sinais cerebelares e cefaléia. Além dessas, os últimos dois trabalhos registraram alterações do estado mental com significativa frequência.

Houve clara predominância do surgimento de lesões no compartimento supratentorial, seguido pelo infratentorial. Naquele houve predominância de lesões nos hemisférios cerebrais, especialmente nos lobos frontais e parietais. No compartimento infratentorial, o acometimento ocorre principalmente no cerebelo. Entre os casos medulares, há predominância de lesões nos segmentos torácicos, seguidas pelas cervicais. Essa observação indica que a disseminação da doença se dê principalmente por via hematogênica, em razão da predominância de lesões em áreas com alto fluxo sanguíneo, especialmente os territórios das artérias cerebrais anterior e média, ramos terminais da artéria carótida interna. Desse modo, o fungo deve estabelecer uma relação com a barreira hematoencefálica, penetrando-a através de algum mecanismo ainda não descrito. Esse achado está de acordo com dados necroscópicos de estudos prévios ${ }^{6365} 66$

A forma de definição do diagnóstico confirmou a pequena importância atribuída à análise do líquor, que se apresenta, geralmente, límpido e com pressão normal ${ }^{50}$. Pode, no entanto, exibir alterações inespecíficas como leve pleocitose, proteínas normais ou aumentadas, atingindo valores de $200 \mathrm{mg} / \mathrm{dL}$, glicorraquia normal ou diminuída e cloretos normais. Raramente, 0 agente pode ser isolado a partir do líquor.

É notável, por outro lado, o papel dos métodos de imagem na abordagem desses pacientes. Pela TC, os granulomas paracoccidioidomicóticos apresentam-se como lesões arredondadas, de localização variável, sem sinais de fomação ou de destruição ósseas, com edema perifocal discreto a intenso, discreto efeito de massa e captação de contraste em anel (somente na periferia) devendo ser feito diagnóstico diferencial com abscessos, gliomas, metástases e, mais raramente, meningiomas, infartos ou hematomas em resoluçã $0^{31}$. Já pela RNM, as lesões são caracterizadas por iso ou hiposinal em T1, hiposinal em T2, com edema periférico e realce nodular ou em anel após a administração de contraste ${ }^{46}$.

Uma grande parcela dos pacientes com a forma neurológica da doença apresentou quadro pulmonar simultâneo, inclusive com alterações à radiografia de tórax. Esses dados encontram-se 
de acordo com as observações de Marchiori e cols ${ }^{47}$, reforçando o papel da radiografia de tórax na abordagem diagnóstica desses pacientes.

Observou-se predominância de pacientes com a forma crônica da doença em sua apresentação pseudotumoral, fato que explicaria o quadro sintomatológico compatível com processo expansivo intracraniano anteriormente descrito, ou seja, sinais de hipertensão intracraniana.

Com relação ao tratamento, houve predominância da utilização de sulfamidas, especialmente da associação sulfametoxazoltrimetoprim, fato que se encontra de acordo com as orientações gerais para o tratamento da NPCM. Observa-se, também, o significativo volume de procedimentos cirúrgicos, muitas vezes indicados em razão da incapacidade de definição prévia do diagnóstico, só realizada após a realização do procedimento.

A análise do desfecho dos quadros mostrou elevado índice de mortalidade. Além disso, a frequência de permanência de seqüelas após a resolução dos casos mostrou-se bastante elevada, com predominância de alterações em funções motoras.

Em conclusão, o presente trabalho realizou um amplo levantamento das informações disponíveis sobre 0 comprometimento neurológico da paracoccidioidomicose a partir de 257 casos observados desde 1919 até 2007. Foram analisados aspectos epidemiológicos, clínicos, diagnósticos e terapêuticos da doença. A elevada mortalidade e, principalmente, a importante determinação de sequielas nos pacientes sobreviventes alertam para o fato de que a NPCM deve ser sempre considerada no diagnóstico diferencial de processos expansivos e/ou meningoencefalíticos do sistema nervoso central a fim de se estabelecer o tratamento precoce e evitar o surgimento de seqüelas incapacitantes.

\section{REFERÊNCIAS}

1. Almeida SM, Queiroz-Telles F, Doi EM, Ono M, Werneck LC. Anti-gp43 antibodies in the cerebrospinal fluid of patients with central nervous system involvement by paracoccidioidomycosis. American Journal of Clinical Pathology 118: 864-868, 2002.

2. Almeida SM, Queiroz-Telles F, Teive HAG, Ribeiro CEL, Werneck LC. Central nervous system paracoccidioidomycosis: clinical features and laboratorial findings. The Journal of Infection 48:193-198, 2004.

3. Almeida SM, Rebelatto CLK, Queiroz-Telles F, Werneck LC. Major histocompatibility complex and central nervous system involvement by paracoccidioidomycosis. The Journal of Infection 51:140-143, 2005.

4. Araújo JC, Werneck L, Cravo MA. South american blastomycosis presentig as a posterior fossa tumor. Journal of Neurosurgery 49: 425-428, 1978.

5. Argollo A, Reis VLL, Niemeyer P, Vuono E. Central nervous system involvement in south american blastomycosis. Transactions of the Royal Society of Tropical Medicine and Hygiene 72: 37-39, 1978

6. Aun RA. Blastomicose do cerebelo: forma tumoral. Arquivos do Hospital da Santa Casa de São Paulo 3: 63-70, 1957.

7. Azevedo AP. Lesões do sistema nervoso central na doença de Lutz (blastomicose brasileira). 0 Hospital 36: 21-49, 1949.

8. Braga FM, Okamura M. Blastomicose medular: apresentação de um caso cirúrgico. Seara Médica Neurocirúrgica 1: 435-441, 1973.

9. Brandão CBPA, Baldi J, Stroppa AT, Grandi UJA, Franco GM, Bara C, Hullack KA. Blastomicose sul-americana: abscesso cerebral e forma pulmonar tumoral. $\mathrm{HE}$ Revista 1: 137-141, 1974.
10. Campos EC, Cademartori MS. Blastomicose mesencefálica. Neurobiologia 26: 83-102, 1963.

11. Campos EP, Bonasser F, Cerutti Jr C, Salles PFC. Itraconazole and fluconazole in three neuro-paracoccidioidomycosis patients. A Folha Médica 112: 173-176, 1996.

12. Casiello A, Klass R. A proposito de uma blastomicosis paracoccidioide a forma de granulia pulmonar y meningea. Revista Médica de Rosário 37: 748-768, 1947.

13. Colli BO, Assiratti Jr JA, Machado HR, Figueiredo JFC, Chimelli L, Salvarani CP, Santos F. Intramedullary spinal cord paracoccidioidomycosis. Arquivos de Neuropsiquiatria 54: 466-473, 1996.

14. Corrêa RB, Puccioni-Sohler M, Artemenko SRT, Nogueira SA, Mattos JP, Novis SAP. Apresentação neurológica incomum no curso de paracoccidioidomicose: relato de um caso. Arquivos de Neuropsiquiatria 49: 456-459, 1991.

15. Coutinho ZF, Silva D, Lazera M, Petri V, Oliveira RM, Sabroza PC, Wanke B. Paracoccidioidomycosis mortality in Brazil (1980-1995). Cadernos de Saúde Pública 18: 1441-1454, 2002.

16. Cunha JCP, Magaldi C, Barros C. Localização nervosa da blastomicose sulamericana. Revista Brasileira de Medicina 12: 702-706, 1955.

17. Dantas AM, Yamane R, Camara AG. South american blastomycosis: ophthalmic and oculomotor nerve lesions. American Journal of Tropical Medicine and Hygiene 43: 386-388, 1990 .

18. del Negro G, Melo e Albuquerque FJ, Campos EP. Localização nervosa da blastomicose sul-americana. Revista do Hospital das Clínicas 9: 64-80, 1954.

19. DuarteALWP, Baruffa G, TerraHBG, RenckDV, PetrucciDMC. Paracoccidioidomicose sistêmica com envolvimento do sistema nervoso central. Revista da Sociedade Brasileira de Medicina Tropical 32: 439-442, 1999.

20. Duarte RD, Maia AL, Duarte JA, Furtado CD. Paracoccidioidomicose cerebral: relato de um caso e revisão de literatura. Revista Pesquisa Médica 31: 37-41, 1997.

21. Elias Jr J, Santos AC, Carlotti Jr CG, Colli B0, Canheu A, Matias C, Furlanetti L, Martinez R, Takayanagui OM, Sakamoto AC, Serafini LN, Chimelli L. Central nervous system paracoccidioidomycosis: diagnosis and treatment. Surgical Neurology 63: S1:13-S1:21, 2005.

22. Fagundes-Pereyra WJ, Carvalho GTC, Góes AM, Lima e Silva FC, Sousa AA. Paracoccidioidomicose do sistema nervoso central: análise de 13 casos. Arquivos de Neuropsiquiatria 64: 269-276, 2006.

23. Farage Filho M, Braga MRG, Kuhn MLS. Granuloma blastomicótico na medula cervical: registro de um caso. Arquivos de Neuropsiquiatria 35: 151-155, 1977.

24. Fava Netto C. Contribuição para o estudo imunológico da blastomicose de Lutz (blastomicose sul-americana). Revista do Instituto Adolfo Lutz 21: 99-194, 1961.

25. Fialho A. Um caso de localização cerebral da micose de Lutz. Jornal Brasileiro de Neurologia 1: 378-383, 1949.

26. Finamor LP, Muccioli C, Martins MC, Rizzo LV, Belfort Jr R. Ocular and central nervous system Paracoccidioidomycosis in a pregnant woman with acquired immunodeficiency syndrome. American Journal of Ophthalmology 134: 456-459, 2002.

27. Forjaz MHH, Fischman 0, Camargo ZP, Vieira Filho JPB, Colombo AL. Paracoccidioidomicose em índios brasileiros da tribo Suruí: estudo clínicolaboratorial de 2 casos. Revista da Sociedade Brasileira de Medicina Tropical 32: 571-575, 1999.

28. França Jr MC, Castro R, Balthazar MLF, Faria AV, Cendes F. Focal status epilepticus as the first manifestation of Paracoccidioidomycosis. European Journal of Neurology 12: 73-74, 2005.

29. Franco GM, Hallack KA, Souza SF, Stroppa ALPC, Hadad DJ. Granuloma de cerebelo: relato de um caso. HU Revista 12: 51-60, 1985.

30. Franco M, Mendes RP, Moscardi-Bacchi M, Rezkallah-Iwasso M, Montenegro MR. Paracoccidioidomycosis. Baillière's Clinical Tropical Medicine and Communicable Diseases 4: 185-220, 1989.

31. Gasparetto EL, Liu CB, Carvalho Neto A, Rogacheski E. Central nervous system paracoccidioidomycosis: imaging findings in 17 cases. Journal of Computer Assisted Tomography 27:12-17, 2003.

32. Gonzales G, Boggino J. Para la casuistica de las formas meningo-encefalicas de la enfermedad de Lutz-Splendore-Almeida (granuloma paracoccidioidico). Anales de la Facultad de Ciencias Médicas (Asunción) 4: 66-78, 1944. 
33. Guerreiro CAM, Chuluc SSD, Branchini MLN. A new treatment for large cerebral paracoccidioidomycosis. Arquivos de Neuropsiquiatria 45: 419-423, 1987.

34. Gurgel N. Blastomycose generalizada. Brazil-Médico 34: 540-541, 1920.

35. Guzmán JR. Paracoccidioidosis del sistema nervioso central: presentación de dos casos. Investigación Clínica 15: 87-101, 1965.

36. Hutzler RU, Brussi MLP, Capitani CM, Lima SS. Acometimento neurológico da paracoccidioidomicose avaliado pela tomografia computadorizada de crânio. Revista Paulista de Medicina 103: 243-244, 1985.

37. Invernici LA, Buone MR, Riegger A, Lorencette NA. Pseudotumores cerebrais por paracoccidioidomicose. Jornal Brasileiro de Neurocirurgia 15: 119-122, 2004.

38. Inza MA, Negroni R, Monteverde DA, Lucatelli N, Andersson A. Paracoccidioidomicosis com localización restringida al sistema nervioso. Revista Neurológica Argentina 11: 71-77, 1985.

39. Lambertucci JR, Lana-Peixoto MA, Pitella JEH. Paracoccidioidomicose do sistema nervoso central. Revista da Sociedade Brasileira de Medicina Tropical 34: 395396, 2001.

40. Leal Filho MB, Borges G, Silva Jr GR, Aguiar AAX, Almeida BR, Vieira MACS, Pinheiro LMR. Paracoccidioidomicose em hemisfério cerebral e tronco encefálico: relato de caso. Arquivos de Neuropsiquiatria 64: 686-689, 2006.

41. Lemmi 0, Pimenta AM. Granuloma paracoccidióidico cerebral: a propósito de um caso operado. Arquivos de Neuropsiquiatria 18: 58-63, 1960.

42. Levy JA, Dillon N, Menezes Neto JR, Sampaio SAP. Meningite na blastomicose sul-americana. Revista Paulista de Medicina 55: 518-520, 1959.

43. Lorenzoni PJ, Chang MR, Paniago AMM, Salgado PR. Meningite paracoccidioidomicótica: relato de caso. Arquivos de Neuropsiquiatria 60:1015-1018, 2002.

44. Machado Filho J, Miranda JL. Considerações relativas à blastomicose sulamericana: localizações, sintomas iniciais, vias de penetração e disseminação em 313 casos consecutivos. O Hospital 58: 129-171, 1960.

45. Maffei WE. Micoses do sistema nervoso. Anais da Faculdade de Medicina de São Paulo 29: 297-322, 1943.

46. Magalhães ACA, Bacheschi LA, Caramelli P, Lo LSS, Menezes Neto JR, ShikanaiYasuda MA, Magalhães A. Paracoccidioidomicose do sistema nervoso central: estudo de cinco casos por ressonância magnética. Revista do Hospital das Clínicas da Faculdade de Medicina de São Paulo 48: 94-97, 1993.

47. Marchiori E, Freitas MAL, Lima RAM. Paracoccidioidomicose medular: relato de um caso. Arquivos de Neuropsiquiatria 47: 224-229, 1989.

48. McEwen JG, Garcia AM, Ortiz BL, Botero S, Restrepo A. In search of the natural habitat of Paracoccidioides brasiliensis. Archives of Medical Research 26: 305-306, 1995

49. Mendes RP. Paracoccidioidomicose (Blastomicose Sul-Americana). In: Veronesi R, Focaccia R (eds) Tratado de Infectologia, $3^{a}$ edição, Editora Atheneu, São Paulo, p. 1383-1390, 2005.

50. Minguetti G. Tomografia computadorizada dos granulomas blastomicóticos encefálicos. Revista do Instituto de Medicina Tropical de São Paulo 25: 99-107, 1983 .

51. Morato-Fernandez RN, Beraldo PSS, Marini M, Costa PHC. Paracoccidioidomicose de localização intramedular e cerebral. Arquivos de Neuropsiquiatria 49: 192197, 1991.

52. Moura LP, Raffin CN, del Negro GMB, Ferreira MS. Paracoccidioidomicose evidenciando comprometimento medular tratada com sucesso por fluconazol. Arquivos de Neuropsiquiatria 52: 82-86, 1994.

53. Negroni R, Helou S. Problemas clínicos em micologia médica: problema $\mathrm{n}^{\circ} 3$. Revista Iberoamericana de Micología 20: 31-33, 2003.

54. Nóbrega JPS, Spina-França A. Neuroparacoccidioidomycosis. In: Franco M, Lacaz CS, Restrepo-Moreno A, del Negro G (ed) Paracoccidioidomycosis, $1^{a}$ edição, CRC Press, Boca Raton, p. 321-330, 1994.

55. Nohmi N, Rocha F, Fava Netto C. Paracoccidioidomicose de localização cerebral. Revista da Associação Médica de Minas Gerais 32: 38-40, 1981.

56. Noleto PA, Lima DB, Pinto JR, Soares MS, Montagna N, Lacerda PRS. Paracoccidioidomicose: diagnóstico diferencial com tumor cerebral. Ars Cvrandi 16: $88-96,1983$
57. Oliveira HA, Almeida ALG, Lyro AS, Oliveira IS. Blastomicose cerebral: forma tumoral. Revista Médica do HSE 23: 247-248, 1971.

58. Pacheco RAB, Arruda WA, Hunhevicz SC, Tsubouchi MH, Torres LFB. Thoracic intraspinal paracoccidioidomycosis. Arquivos de Neuropsiquiatria 54: 474-478, 1996.

59. Paglioli E, Tibiriçá PQT, Becker PFL. Micoses do sistema nervoso: estudo de dois casos. Anais da Faculdade de Medicina de Porto Alegre 2: 171-182, 1951.

60. Paniago AM, Oliveira PA, Aguiar ES, Aguiar JI, Cunha RV, Leme LM, Salgado PR, Domingos JA, Ferraz RL, Chang MR, Bóia MN, Wanke B. Neuroparacoccidioidomycosis: analysis of 13 cases observed in an endemic area in Brazil. Transactions of the Royal Society of Tropical Medicine and Hygiene 101:414-420, 2007.

61. Pedro RJ, Branchini MLM, Lucca RS, Silveira ML, Facure NO, Amato Neto V. Paracoccidioidomicose de sistema nervoso central: a propósito de dois casos. Revista do Instituto de Medicina Tropical de São Paulo 22: 269-274, 1980.

62. Pedroso VSP, Vilela MC, Pedroso ERP, Teixeira AL. Paracoccidioidomicose com comprometimento do sistema nervoso central: revisão de literatura. Revista Brasileira de Neurologia 44: 33-40, 2008.

63. Peña CE. Compromiso del sistema nervioso central em blastomicosis suramericana. Mycologia Applicata 33: 280-288, 1967.

64. Pereira JM, Jacobs F. Um caso de blastomycose cutânea com accesos epilépticos. Annaes Paulista de Medicina e Cirurgia 10: 217-219, 1919.

65. Pereira WC, Raphael A, Sallum J. Lesões neurológicas na blastomicose sulamericana: estudo anatomopatológico de 14 casos. Arquivos de Neuropsiquiatria 23: 95-112, 1965.

66. Pereira WC, Raphael A, Tenuto RA, Sallum J. Localização encefálica da blastomicose sul-americana: considerações a propósito de 9 casos. Arquivos de Neuropsiquiatria 23: 113-126, 1965.

67. Perino FR, Lavarello A, Dimier HG. Blastomicosis sudamericana: granuloma paracoccidioidico de lozalización pulmonar y cerebral. La Prensa Médica Argentina 49: 607-613, 1962.

68. Pimenta AM, Marques JS, Settani FAP. Blastomicose cerebral, forma tumoral. Seara Médica Neurocirúrgica (São Paulo) 1: 73-84,1972.

69. Pinto JP, Silva AQ, Silveira 0. Blastomicose cerebral: forma isolada com sintomatologia de tumor cerebral. Revista Médica do Paraná 28: 57-62, 1959.

70. PláMP,HartungC,MendozaP,StukanoffA,MorenoMJ.Neuroparacoccidioidomycosis: case reports and review. Mycopathologia 127: 139-144, 1994.

71. Prado JM, Insausti T, Matera RF. Contribución al estudio de las coccidio y paracoccidiomicosis del sistema nervioso. Archivos de Neurocirurgía 3: 90-106, 1946.

72. Raphael A, Pereira WC. Granuloma blastomicótico cerebral: relato de um caso tratado cirùrgicamente e com anfotericina $\mathrm{B}$. Revista do Hospital das Clínicas 17: 430-433, 1962.

73. Raphael A. Localização nervosa da blastomicose sul-americana. Arquivos de Neuropsiquiatria 24: 69-90, 1966.

74. Reis BS, Bozzi A, Prado FLS, Pereira MCN, Ferreira FE, Godoy P, Moro L, Pedroso EP, Leite MF, Goes AM. Membrane and extracellular antigens of Paracoccidioides brasiliensis (Mexo): Identification of a $28-\mathrm{kD}$ a protein suitable for immunodiagnosis of paracoccidioidomycosis. Journal of Immunological Methods 307: 118-126, 2005.

75. Ritter FH. Tumor cerebral granulomatoso por paracoccidióide: a propósito de dois casos operados. Arquivos de Neuropsiquiatria 6: 352-359, 1948.

76. Rocca ED, Franco J. Granuloma micótico cerebral. Revista de Neuropsiquiatria 20: 107-110, 1957.

77. Rodacki MA, Toni G, Borba LA, Oliveira GG. Paracoccidioidomycosis of the central nervous system: CT findings. Neuroradiology 37: 636-641, 1995.

78. Sammartino R. Absceso cerebeloso por Paracoccidioides brasiliensis. Archivos de La Sociedad Argentina de Anatomia Normal y Patológica 9: 360-367, 1947.

79. Saravia JG, Restrepo MT, Toro GG, Vergara IG. Paracoccidioidomicosis del sistema nervioso central. Revista Facultad de Medicina de la Universidad Nacional de Colombia 39: 27-37, 1973

80. Silva CEAP, Cordeiro AF, Gollner AM, Cupolilo SMN, Quesado-Filgueiras M, Curzio MF. Paracoccidioidomicose do sistema nervoso central: relato de caso. Arquivos de Neuropsiquiatria 58: 741-747, 2000. 
81. Silva SHM, Colombo AL, Blotta MHSL, Lopes JD, Queiroz-Telles F, Camargo ZP. Detection of circulating gp43 antigen in serum, cerebrospinal fluid and bronchoalveolar lavage fluid of patients with paracoccidioidomycosis. Journal of Clinical Microbiology 41: 3675-3680, 2003.

82. Silva SHM, Grosso DM, Lopes JD, Colombo AL, Blotta MHSL, Queiroz-Telles F, Camargo ZP. Detection of Paracoccidioides brasiliensis gp 70 circulating antigen and follow-up of patients undergoing antimycotic therapy. Journal of Clinical Microbiology 42: 4480-4486, 2004.

83. Sousa MR, Xavier AJ, Vieira NRN, Pereira CML, Condak CE, Carmo JA, Pedroso ERP, Souza AH, Oliveira JT. Paracoccidioidomicose crônica disseminada com caquexia, lesão pulmonar grave e granuloma cerebral em paciente sem imunodeficiência adquirida. Revista Médica de Minas Gerais 5: 257-260, 1995.

84. Teive HAG, Arruda WO, Ramina R, Meneses MS, Bleggi-Torres LF, Telles-Filho FQ. Paracoccidioidomycosis granuloma simulating posterior fossa tumour. Journal of the Royal Society of Medicine 84: 562-563, 1991.
85. Tristano AG, Chollet ME, Willson M, Perez J, Troccoli M. Central nervous system Paracoccidioidomycosis: case report and review. Investigación Clínica 45: 277-288, 2004

86. Turovetzky A, Koren F, Negroni R, Grün D, Stortini R. Lesión expansiva en cerebelo por Paracoccidioides brasiliensis. Revista Argentina de Micología 3: 9-12, 1980

87. Valle ACF, Skacel M, Costa RLB, Ribeiro CT, Montagna NAR, Cruz LCH. A case of intraspinal Paracoccidioidomycosis. Revista do Instituto de Medicina Tropical de São Paulo 40: 203-207, 1998.

88. Villa LA, Tobón A, Restreppo A, Calle D, Rosero DS, Gómez BL, Restrepo A Central nervous system paracoccidioidomycosis: report of a case successfully treated with itraconazole. Revista do Instituto de Medicina Tropical de São Paulo 42: 231-234, 2000

89. Wittig EO, Kasting G, Leal R. Neuroblastomicose: registro de três casos. Arquivos de Neuropsiquiatria 26: 73-79, 1968. 